\title{
ADMISSIBLE SOLUTIONS OF THE SCHWARZIAN DIFFERENTIAL EQUATION
}

\author{
KATSUYA ISHIZAKI
}

(Received 29 March 1989; revised 12 September 1989)

\begin{abstract} the Schwarzian equation

$$
\{w, z\}^{m}=R(z, w)
$$
forms:

$$
\begin{aligned}
\{u, z\} & =C \frac{\left(u-\sigma_{1}\right)\left(u-\sigma_{2}\right)\left(u=\sigma_{3}\right)\left(u-\sigma_{4}\right)}{\left(u-\tau_{1}\right)\left(u-\tau_{2}\right)\left(u-\tau_{3}\right)\left(u-\tau_{4}\right)}, \\
\{u, z\}^{3} & =C \frac{\left[\left(u-\sigma_{1}\right)^{3}\left(u-\sigma_{2}\right)^{3}\right]}{\left[\left(u-\tau_{1}\right)^{3}\left(u-\tau_{2}\right)^{2}\left(u-\tau_{3}\right)\right]}, \\
\{u, z\}^{3} & =C \frac{\left[\left(u-\sigma_{1}\right)^{3}\left(u-\sigma_{2}\right)^{3}\right]}{\left[\left(u-\tau_{1}\right)^{2}\left(u-\tau_{2}\right)^{2}\left(u-\tau_{3}\right)^{2}\right]}, \\
\{u, z\}^{2} & =C \frac{\left[\left(u-\sigma_{1}\right)^{2}\left(u-\sigma_{2}\right)^{2}\right]}{\left[\left(u-\tau_{1}\right)^{2}\left(u-\tau_{2}\right)\left(u-\tau_{3}\right)\right]}, \\
\{u, z\} & =C \frac{\left[\left(u-\sigma_{1}\right)\left(u-\sigma_{2}\right)\right]}{\left[\left(u-\tau_{1}\right)\left(u-\tau_{2}\right)\right]}, \\
\{u, z\} & =C,
\end{aligned}
$$
\end{abstract}

Let $R(z, w)$ be a rational function of $w$ with meromorphic coefficients. It is shown that if

possesses an admissible solution, then $d+2 m \sum_{j=1}^{l} \delta\left(\alpha_{j}, w\right) \leq 4 m$, where $\alpha_{j}$ are distinct complex constants. In particular, when $R(z, w)$ is independent of $z$, it is shown that if (*) possesses an admissible solution $w(z)$, then by some Möbius transformation $u=$ $(a w+b) /(c w+d) \quad(a d-b c \neq 0)$, the equation can be reduced to one of the following

where $\tau_{j}(j=1, \ldots, 4)$ are distinct constants, and $\sigma_{j}(j=1, \ldots, 4)$ are constants, not necessarily distinct.

1980 Mathematics subject classification (Amer. Math. Soc.) (1985 Revision): primary 34 C 10; secondary 30 D 35.

Keywords and phrases: admissible solution, Schwarzian, Nevanlinna theory.

(C) 1991 Australian Mathematical Society 0263-6115/91 \$A2.00+0.00 


\section{Introduction}

Let $w(z)$ be a meromorphic function, and $\{w, z\}$ be its Schwarzian derivative:

$$
\{w, z\}=\left(\frac{w^{\prime \prime}}{w^{\prime}}\right)^{\prime}-\frac{1}{2}\left(\frac{w^{\prime \prime}}{w^{\prime}}\right)^{2}
$$

Here we consider the differential equation

$$
\{w, z\}^{m}=R(z, w)=P(z, w) / Q(z, w),
$$

where $P(z, w)$ and $Q(z, w)$ are polynomials of $w$ with meromorphic coefficients, with $\operatorname{deg}_{w}[P(z, w)]=p$ and $\operatorname{deg}_{w}[Q(z, w)]=q$, respectively:

$$
\left(1.1^{\prime}\right) \begin{cases}P(z, w)=\xi_{p}(z) w^{p}+\xi_{p-1}(z) w^{p-1}+\cdots+\xi_{0}(z), & \xi_{p}(z) \not \equiv 0, \\ Q(z, w)=\eta_{q}(z) w^{q}+\eta_{q-1}(z) w^{q-1}+\cdots+\eta_{0}(z), & \eta_{q}(z) \not \equiv 0\end{cases}
$$

where $\xi_{j}(z), \eta_{k}(z)$ are meromorphic functions. We suppose that $P(z, w)$ and $Q(z, w)$ are mutually prime. Sometimes we call

$$
\left(1.1^{\prime \prime}\right) \quad \xi_{j}(z) / \eta_{q}(z) \text { and } \eta_{k}(z) / \eta_{q}(z)
$$

the reduced coefficients of $R(z, w)$. Put

$$
\max (p, q)=\operatorname{deg}_{w}[R(z, w)]=d .
$$

We are concerned with the determination of the equations (1.1) which admit transcendental meromorphic solutions.

Steinmetz [11] treated the case $m=1$ and $d=0$ in (1.1), and the present author [4] investigated the case $m=1$ and $d \geq 0$. Here we will consider the case $m \geq 1$ and $d \geq 0$.

We use standard notations in Nevanlinna theory.

Let $f(z)$ be a meromorphic function. As usual, $m(r, f), N(r, f)$ and $T(r, f)=m(r, f)+N(r, f)$ denote the proximity function, the counting function, and the characteristic function of $f(z)$, respectively. For $\alpha \in \mathbb{C}$, put

$$
m(r, \alpha ; f)=m(r, 1 /(f-\alpha)), \quad N(r, \alpha ; f)=N(r, 1 /(f-\alpha)) .
$$

Sometimes, we write $m(r, f)$ or $N(r, f)$ as $m(r, \infty ; f)$ and $N(r, \infty ; f)$. 
Let $\bar{n}(r, f)$ be the number of distinct poles of $f(z)$ in $|z| \leq r$, and put

$$
\begin{gathered}
\bar{N}(r, f)=\int_{0}^{r} \frac{\bar{n}(t, f)-\bar{n}(0, f)}{t} d t+\bar{n}(0, f) \log r, \\
\bar{N}(r, \alpha ; f)=\bar{N}(r, 1 /(f-\alpha)), \quad \alpha \in \mathbb{C}, \\
N_{1}(r, f)=N(r, f)-\bar{N}(r, f), \quad N_{1}(r, \alpha ; f)=N_{1}(r, 1 /(f-\alpha)) .
\end{gathered}
$$

Let $\bar{n}^{*}(r, 0 ; f, g)$ be the number of distinct common zeros of $f(z)$ and $g(z)$ in $|z| \leq r$, and put

$$
\begin{aligned}
\bar{N}^{*}(r, 0 ; f, g)= & \int_{0}^{r} \frac{\bar{n}^{*}(t, 0 ; f, g)-\bar{n}^{*}(0,0 ; f, g)}{t} d t \\
& +n^{*}(0,0 ; f, g) \log r .
\end{aligned}
$$

Further we put as usual $[9$, pages $226,277,280]$

$$
\begin{array}{rlr}
\delta(\alpha, f) & =\varliminf_{r \rightarrow \infty} \frac{m(r, \alpha ; f)}{T(r, f)}=1-\varlimsup_{r \rightarrow \infty} \frac{N(r, \alpha ; f)}{T(r, f)} & \text { (deficiency), } \\
\theta(\alpha, f) & =\varliminf_{r \rightarrow \infty} \frac{N_{1}(r, f)}{T(r, f)} & \text { (ramification index), } \\
\Theta(\alpha, f) & =\varliminf_{r \rightarrow \infty} \frac{m(r, \alpha ; f)+N_{1}(r, \alpha ; f)}{T(r, f)} & \text { (total ramification). }
\end{array}
$$

A function $\varphi(r), 0 \leq r<\infty$, is said to be $S(r, f)$ if there is a set $E \subset \mathbb{R}^{+}$ of finite linear measure such that

$$
\varphi(r)=o(T(r, f)) \text { as } r \rightarrow \infty, r \notin E .
$$

A meromorphic function function $a(z)$ is called small with respect to $f(z)$, if $T(r, a)=S(r, f)$.

Let $a_{1}(z), \ldots, a_{n}(z)$ be meromorphic functions. A transcendental meromorphic function $w(z)$ is called admissible with respect to $a_{j}(z)$. If

$$
T\left(r, a_{j}\right)=S(r, w), \quad j=1, \ldots, n .
$$

We call $w(z)$ an admissible solution of (1.1), if $w(z)$ satisfies (1.1) and is admissible with respect to the reduced coefficients of $R(z, w)$ (see $\left(1.1^{\prime \prime}\right)$ ). In this paper, "admissible" implies "transcendental".

RemarK 1. Suppose (1.1) possesses an admissible solution $w=w(z)$. Then we have $\bar{N}^{*}(r, 0 ; P, Q)=S(r, w)$, where $P(z)=P(z, w(z))$ and $Q(z)=Q(z, w(z))$. For, since $P(z, w)$ and $Q(z, w)$ are mutually prime, there exist polynomials of $w, U(z, w)$ and $V(z, w)$ such that

$$
U(z, w) P(z, w)+V(z, w) Q(z, w)=s_{P, Q}(z)=s(z),
$$

where $s(z)$ and coefficients of $U(z, w)$ and $V(z, w)$ are small functions 
with respect to $w(z)$. Suppose $\bar{N}(r, 0 ; P, Q) \neq S(r, w)$. Then $N(r, 1 / s)$ $\neq S(r, w)$, which is a contradiction. Hence if $z_{0}$ is a zero of $Q(z, w(z))$ which is neither a zero nor a pole of $s(z)$ or of the coefficients of $P(z, w)$ and $Q(z, w)$, then $z_{0}$ is a pole of $R(z)=P(z, w(z)) / Q(z, w(z))$ (see [5, page 169]).

Our results are as follows:

TheOREM 1. Let $\alpha_{1}, \alpha_{2}, \ldots, \alpha_{l}$ be distinct constants. If $(1.1)$ possesses an admissible solution, then we have

$$
d+2 m \sum_{j=1}^{l} \delta\left(\alpha_{j}, w\right) \leq 4 m .
$$

REMARK 2. Inequality (1.3) is a limitation for $d=\operatorname{deg}[R]$ by deficiencies of a solution. Mues [8] classified the algebraic Riccati equations by the number of Picard exceptional values of transcendental solutions. In this connection we classified [4] the Riccati equations (with meromorphic coefficients) by the number of Picard exceptional values of admissible solutions. We showed that, if $w(z)$ satisfies a Riccati equation, then $w(z)$ also satisfies a Schwarzian differential equation (1.1) with $m=1$ for some $R(z, w)$, and that, if $w(z)$ has $l(=1$ or 2) Picard exceptional values, then $\operatorname{deg}_{w}[R(z, w)]=4-2 l$. Further, if $w(z)$ has no Picard value, then $\operatorname{deg}_{w}[R(z, w)]=2$ or 4 . Theorem 1 is a generalization of these results.

THEOREM 2. If (1.1) possesses an admissible solution, then the denominator $Q(z, w)$ of $R(z, w)$ must be one of the following:

$$
\begin{aligned}
& Q(z, w)=c(z)\left(w+b_{1}(z)\right)^{2 m}\left(w+b_{2}(z)\right)^{2 m}, \\
& Q(z, w)=c(z)\left(w^{2}+a_{1}(z) w+a_{0}(z)\right)^{2 m}, \\
& Q(z, w)=c(z)(w+b(z))^{2 m}, \\
& Q(z, w)=c(z)(w+b(z))^{2 m}\left(w-\tau_{1}\right)^{m}\left(w-\tau_{2}\right)^{m}, \\
& Q(z, w)=c(z)(w+b(z))^{2 m}\left(w-\tau_{1}\right)^{2 m / n}, \quad n \mid(2 m), n \geq 2,
\end{aligned}
$$$$
Q(z, w)=c(z)\left(w-\tau_{1}\right)^{m}\left(w-\tau_{2}\right)^{m}\left(w-\tau_{3}\right)^{m}\left(w-\tau_{4}\right)^{m}
$$$$
Q(z, w)=c(z)\left(w-\tau_{1}\right)^{m}\left(w-\tau_{2}\right)^{m}\left(w-\tau_{3}\right)^{2 m / n},
$$$$
n \mid(2 m), n \geq 2,
$$ 


$$
\begin{aligned}
& Q(z, w)=c(z)\left(w-\tau_{1}\right)^{m}\left(w-\tau_{2}\right)^{2 m / 3}\left(w-\tau_{3}\right)^{2 m / 6}, \\
& Q(z, w)=c(z)\left(w-\tau_{1}\right)^{2 m / 3}\left(w-\tau_{2}\right)^{2 m / 3}\left(w-\tau_{3}\right)^{2 m / 3}, \\
& Q(z, w)=c(z)\left(w-\tau_{1}\right)^{m}\left(w-\tau_{2}\right)^{2 m / 4}\left(w-\tau_{3}\right)^{2 m / 4}, \\
& Q(z, w)=c(z)\left(w-\tau_{1}\right)^{2 m / n_{1}}\left(w-\tau_{2}\right)^{2 m / n_{2}}, \quad n_{j} \mid(2 m), n_{j} \geq 2, \\
& Q(z, w)=c(z)\left(w-\tau_{1}\right)^{2 m / n}, \quad n \mid 2 m, n \geq 2, \\
& Q(z, w)=c(z),
\end{aligned}
$$

where $c(z), a_{1}(z), a_{0}(z)$ are meromorphic functions, $\left|a_{1}^{\prime}\right|+\left|a_{2}^{\prime}\right| \neq 0, b_{1}(z)$, $b_{2}(z), b(z)$ are nonconstant meromorphic functions, and $\tau_{j}(j=1,2,3,4)$ are distinct constants.

In particular, if $R(z, w)$ in (1.1) is independent of $z$, then we have

$$
\{w, z\}^{m}=P(w) / Q(w)=\left(w-\sigma_{1}\right)^{\lambda_{1}} \cdots\left(w-\sigma_{n}\right)^{\lambda_{h}} / Q(w),
$$

where $\sigma_{j}(j=1, \ldots, h)$ are distinct constants, and $Q(w)$ is one of the polynomials $(1.9)-(1.19)$, with $c(z)$ constant.

THEOREM 3. Suppose, in (1.1), that $R(z, w)$ is independent of $z$. If $(1.20)$ possesses an admissible solution $w(z)$, then by some Möbius transformation $u=(a w+b) /(c w+d), a d-b c \neq 0$, the equation can be reduced to one of the following forms:

$$
\begin{aligned}
\{u, z\} & =C \frac{\left(u\left[\sigma_{1}\right)\left(u-\sigma_{2}\right)\left(u-\sigma_{3}\right)\left(u-\sigma_{4}\right)\right.}{\left(u-\tau_{1}\right)\left(u-\tau_{2}\right)\left(u-\tau_{3}\right)\left(u-\tau_{4}\right)}, \\
\{u, z\} & =C \frac{\left[\left(u-\sigma_{1}\right)^{3}\left(u-\sigma_{2}\right)^{3}\right]}{\left[\left(u-\tau_{1}\right)^{3}\left(u-\tau_{2}\right)^{2}\left(u-\tau_{3}\right)\right]}, \\
\{u, z\}^{3} & =C \frac{\left[\left(u-\sigma_{1}\right)^{3}\left(u-\sigma_{2}\right)^{3}\right]}{\left[\left(u-\tau_{1}\right)^{2}\left(u-\tau_{2}\right)^{2}\left(u-\tau_{3}\right)^{2}\right]}, \\
\{u, z\}^{2} & =C \frac{\left[\left(u-\sigma_{1}\right)^{2}\left(u-\sigma_{2}\right)^{2}\right]}{\left[\left(u-\tau_{1}\right)^{2}\left(u-\tau_{2}\right)\left(u-\tau_{3}\right)\right]}, \\
\{u, z\} & =C \frac{\left[\left(u-\sigma_{1}\right)\left(u-\sigma_{2}\right)\right]}{\left[\left(u-\tau_{1}\right)\left(u-\tau_{2}\right)\right]}, \\
\{u, z\} & =C
\end{aligned}
$$

where $\tau_{j}(j=1, \ldots, 4)$ are distinct constants, and $\sigma_{j}(j=1, \ldots, 4)$ are constants, not necessarily distinct.

The equations (1.21)-(1.26) possess admissible solutions.

We will prove these theorems in Sections $3,4,5$, respectively. 


\section{Preliminary material}

We recall some well-known properties of the Schwarzian derivative [2].

LeMMA A. Let $w(z)$ be a meromorphic function.

(a) If $z_{0}$ is a simple pole of $w(z)$, then $\{w, z\}$ is regular at $z_{0}$.

(b) If $z_{0}$ is a multiple pole of $w(z)$ or a zero of $w^{\prime}(z)$, then $z_{0}$ is a double pole of $\{w, z\}$. Further, if

$$
w(z)=c_{m}\left(z-z_{0}\right)^{-m}+c_{m+1}\left(z-z_{0}\right)^{-m+1}+\cdots
$$

or

$$
w(z)=c_{0}+c_{m}\left(z-z_{0}\right)^{m}+c_{m+1}\left(z-z_{0}\right)^{m+1}+\cdots
$$

with $c_{m} \neq 0, m \geq 2$, in a neighborhood of $z_{0}$, then we have

$$
\{w, z\}=\left[\left(1-m^{2}\right) / 2\right]\left(z-z_{0}\right)^{-2}+\left[\left(m^{2}-1\right) c_{m-1} / m c_{m}\right]\left(z-z_{0}\right)^{-1}+\cdots
$$

or

$$
\{w, z\}=\left[\left(1-m^{2}\right) / 2\right]\left(z-z_{0}\right)^{-2}+\left[\left(1-m^{2}\right) c_{m+1} / m c_{m}\right]\left(z-z_{0}\right)^{-1}+\cdots,
$$

respectively.

(c) $\{L(w), z\}=\{w, z\}$ for any Möbius transformation $L$.

The following theorem was proved in [3].

THEOREM B. Let $f(z)$ be a transcendental meromorphic function and $Q(z, f)$ be a polynomial of $f$ with small meromorphic coefficients with respect to $f$ and $\operatorname{deg}[Q] \leq n-2$. Let $a(z)$ be a small meromorphic function and $F(z)=A(z) f(z)^{n}-Q(z, f(z))$. If $Q(z, f(z)) \neq 0$, then

$$
w T(r, f) \leq \bar{N}(r, f)+\bar{N}(r, 0 ; f)+\bar{N}(r, 0 ; F)+S(r, f) .
$$

Steinmetz and Rieth characterized differential equations of the form (2.2) below, which have admissible solutions:

THEOREM C ([5], [10], [12]). Let $R(z, w)$ be a rational function of $w$ with meromorphic coefficients. Suppose the differential equation

$$
w^{\prime m}=R(z, w)
$$

admits an admissible solution $w=w(z)$. Then, by a Möbius transformation $u=(a w+b) /(c w+d), a d-b c \neq 0,(2.2)$ is reduced to one of the following 
equations:

$$
\begin{aligned}
& u^{\prime}=a(z)+b(z) u+c(z) u^{2} \\
& u^{\prime 2}=a(z)(u-b(z))^{2}\left(u-\tau_{1}\right)\left(u-\tau_{2}\right), \quad b(z) \neq \tau_{1}, \tau_{2} ; \\
& u^{\prime 2}=a(z)\left(u-\tau_{1}\right)\left(u-\tau_{2}\right)\left(u-\tau_{3}\right)\left(u-\tau_{4}\right) ; \\
& u^{\prime 3}=a(z)\left(u-\tau_{1}\right)^{2}\left(u-\tau_{2}\right)^{2}\left(u-\tau_{3}\right)^{2} ; \\
& u^{\prime 4}=a(z)\left(u-\tau_{1}\right)^{2}\left(u-\tau_{2}\right)^{3}\left(u-\tau_{3}\right)^{3} ; \\
& u^{\prime 6}=a(z)\left(u-\tau_{1}\right)^{3}\left(u-\tau_{2}\right)^{4}\left(u-\tau_{3}\right)^{5} ;
\end{aligned}
$$

where $\tau_{j}$ are distinct constants, and $a(z), b(z), c(z)$ are meromorphic.

We further need the following lemmas:

LEMMA 1. Suppose $w=w(z)$ is an admissible solution of (1.1). If we write $Q(z, w(z))$ as $Q(z)$, then

$$
q T(r, w)+S(r, w) \leq N(r, 1 / Q) .
$$

LEMMA 2. Let the polynomial $Q(z, w)$ be factored as follows:

$$
Q(z, w)=c(z)\left(V_{1}(z, w)\right)^{\mu_{1}} \cdots\left(V_{k}(z, w)\right)^{\mu_{k}},
$$

where $c(z)$ is meromorphic and $V_{j}(z, w), j=1, \ldots, k$, are polynomials of $w$ with meromorphic coefficients, irreducible and mutually prime. Suppose (1.1) possesses an admissible solution $w=w(z)$.

(i) If $V_{j_{0}}(z)=\left.\frac{\partial}{\partial z} V_{j_{0}}(z, w)\right|_{w=w(z)} \not \equiv 0$, then $\mu_{j_{0}}=2 \mathrm{~m}$.

(ii) If $V_{j_{1} z}(z)=\left.\frac{\partial}{\partial z} V_{j_{1}}(z, w)\right|_{w=w(z)} \equiv 0$, then $\mu_{j_{1}} \mid(2 m)$ and $\mu_{j_{1}} \leq m$.

Now we consider the case when $R(z, w)$ is independent of $z$ :

$$
\{w, z\}^{m}=P(w) / Q(w)=\left(w-\sigma_{1}\right)^{\lambda_{1}} \cdots\left(W-\sigma_{h}\right)^{\lambda_{h}} / Q(w) .
$$

Without loss of generality, we may assume below that $\operatorname{deg}[P(w)]=$ $\operatorname{deg}[Q(w)]$, by applying a Möbius transformation $L$ to $w$ if necessary.

LEMMA 3. Suppose (1.20) possesses an admissible solution $w(z)$. If $w(z)$ takes the value $\sigma_{1}$, then $m \mid \lambda_{i}$.

LEMMA 4. Suppose (1.20), with $Q(w)$ of the form (1.18), where $c(z)$ is constant, possesses an admissible solution. Then we have $n=2$.

Lemma 5. Suppose (1.20) possesses an admissible solution. Then $m \mid \lambda_{i}$, $i=1, \ldots, h$. 
Lemma 6. Let $C \neq 0, \sigma_{i}, i=1,2,3$, by constants and $\tau_{j}, j=1,2,3$, be distinct constants. Then the differential equation

$$
\{w, z\}=C\left(w-\sigma_{1}\right)\left(w-\sigma_{2}\right)\left(w-\sigma_{3}\right) /\left[\left(w-\tau_{1}\right)\left(w-\tau_{2}\right)\left(w-\tau_{3}\right)\right]
$$

possesses no admissible solution.

LemMa 7. Let $C$ be a nonzero constant and $\sigma, \tau$ be distinct constants. Then the differential equation

$$
\{w, z\}=C(w-\sigma) /(w-\tau)
$$

possesses no admissible solution.

LeMMA 8. Let $C \neq 0, \sigma_{1}$, and $\sigma_{2}$ be constants and $\tau_{1}, \tau_{2}$ be distinct constants. Then the differential equation

$$
\{w, z\}^{2}=C\left(w-\sigma_{1}\right)\left(w-\sigma_{2}\right) /\left[\left(w-\tau_{1}\right)\left(w-\tau_{2}\right)\right]
$$

possesses no admissible solution.

Finally we define a usual symbol $\omega$. For a meromorphic function $g(z)$, we define $\omega\left(z_{0}, g\right)$ as follows: if $z_{0}$ is a pole of order $m(\geq 1)$ for $g(z)$, then $\omega\left(z_{0}, g\right)=m$; if $g\left(z_{0}\right) \neq \infty$, then $\omega\left(z_{0}, g\right)=0$.

\section{Proofs of Lemma 1 and Theorem 1}

Proof of Lemma 1. By the lemma on logarithmic derivatives [5], we have

$$
m(r, R)=m\left(r,\{w, z\}^{m}\right)=S(r, w),
$$

where $R$ denotes $R(z, w(z))$. It is proved in [7] that

$$
d T(r, w)+S(r, w)=T(r, R)=N(r, R)+m(r, R) .
$$

By (3.1) and (3.2), we have

$$
d T(r, w)=N(r, R)+S(r, w) .
$$

If $p>q$ so that $d=p$, then

$$
\begin{aligned}
N(r, R) & \leq(p-q) N(r, w)+N(r, 1 / Q)+S(r, w) \\
& \leq(p-q) T(r, w)+N(r, 1 / Q)+S(r, w),
\end{aligned}
$$

where $Q$ denotes $Q(z, w(z))$. By (3.3) and (3.4), $q T(r, w)+S(r, w) \leq$ $N(r, 1 / Q)$, which proves (2.3) for the case $p>q$. If $p \leq q=d$, then

$$
N(r, R) \leq N(r, 1 / Q)+S(r, w) .
$$


By (3.3) and (3.5), we also obtain (2.3) in the case $p \leq q$.

Proof of Theorem 1. First we consider the case $p \leq q$. By Remark 1 , there exists a small (w.r.t. $w(z)$ ) function $s_{P, Q}(z)=s(z)$ such that common zeros of $P(z, w(z))$ and $Q(z, w(z))$ are zeros of $s(z)$. Let $z_{0}$ be a zero of $Q(z, w(z))$ such that, in $\left(1.1^{\prime}\right), \xi_{j}\left(z_{0}\right) \neq 0, \infty$ and $\eta_{k}\left(z_{0}\right) \neq 0, \infty$ for $0 \leq j \leq p, 0 \leq k \leq q$ and $s\left(z_{0}\right) \neq 0$. By (1.1) and Lemma $\mathrm{A}(\mathrm{b}), z_{0}$ must be a zero of $w^{\prime}(z)$ and hence a double pole of $\{w, z\}$. Thus we have

$$
2 m \bar{N}\left(r, 1 / w^{\prime}\right)=N(r, 1 / Q)+S(r, w) .
$$

Hence

$$
\frac{1}{2 m} N(r, 1 / Q) \leq N\left(r, 1 / w^{\prime}\right)+S(r, s) .
$$

By Lemma 1 and the second fundamental theorem we obtain, using (3.7), that

$$
\frac{d}{2 m} T(r, w)+\sum_{j=1}^{l} m\left(r, \alpha_{j} ; w\right) \leq 2 T(r, w)+S(r, w),
$$

and thus we obtain (1.3) in this case:

$$
d+2 m \sum_{j=1}^{l} \delta\left(\alpha_{j}, w\right) \leq 4 m .
$$

Next, suppose $p>q$. Choose $c \in \mathbb{C}$ such that $Q(z, c) \not \equiv 0$ and put $u=1 /(w-c)$ in (1.1). Then by Lemma $\mathrm{A}(\mathrm{c})$

$$
\{u, z\}^{m}=\frac{\xi_{p}(z)\left(\frac{1}{u}\right)^{p}+, \ldots,+P(z, c)}{\eta_{q}(z)\left(\frac{1}{u}\right)^{q}+, \ldots,+Q(z, c)}=P_{1}(z, u) / Q_{1}(z, u)
$$

and $\operatorname{deg}_{u}\left[Q_{1}(z, u)\right]=d$, and hence we can apply the arguments for the case $p \leq q$ and obtain (3.8) also.

EXAMPLE 1. Suppose $w(z)$ satisfies the Schwarzian differential equation $\{w, z\}=R(z, w)$. By Theorem 1, if $w(z)$ possesses $j$ Picard exceptional values $(j=1,2)$, then $\operatorname{deg}_{w}[R(z, w)] \leq 4-2 j$. Solutions of the equations

$$
\begin{gathered}
w^{\prime}=w^{2}+\alpha w+\beta \quad\left(\alpha, \beta \in \mathbb{C}, \alpha^{2}-4 \beta \neq 0\right), \\
w^{\prime}=(w-\alpha)(w+z) \quad(\alpha \in \mathbb{C}), \\
w^{\prime}=(w+z)^{2}, \\
w^{\prime}=w^{2}+z
\end{gathered}
$$

possess two, one, no and again no Picard exceptional values, respectively, 
and satisfy the corresponding one of the following equations:

$$
\begin{aligned}
& \{w, z\}=2\left(\beta-\alpha^{2} / 4\right), \\
& \{w, z\}=\frac{-\left[\left(4+z^{2}\right) w^{2}+2 z\left(z^{2}+2\right) w+z^{4}+3\right]}{\left[2(w+z)^{2}\right]}, \\
& \{w, z\}=\frac{-4\left[(w+z)^{2}+a\right]}{(w+z)^{2}}, \\
& \{w, z\}=\frac{\left[4 z w^{4}-8 w^{3}+8 z^{2} w^{2}-8 z w+4 z^{3}-3\right]}{\left[\left(2\left(w^{2}+z\right)^{2}\right]\right.} .
\end{aligned}
$$

\section{Proofs of Lemma 2 and Theorem 2}

Proof of Lemma 2. (i) Write $V_{j_{0}}(z, w(z))$ simply as $V(z)$. Since $V(z, w)$ is irreducible, $V(z, w)$ and $V_{z}(z, w)=\partial V(z, w) / \partial z$ are mutually prime as polynomials of $w$. Thus by Remark 1 , there exists a $s_{V, V_{z}}(z)$, which is a small function with respect to $w(z)$, such that $\bar{N}^{*}\left(r, 0 ; V, V_{z}\right) \leq$ $N\left(r, 1 / s_{V, V_{z}}\right) \leq S(r, w)$ (see [5, pages 173-174]).

By Lemma $1, V(z)$ has infinitely many zeros and $m(r, 0 ; V)=S(r, w)$. By Remark $1, \bar{N}^{*}(r, 0 ; P, V) \leq N\left(r, 1 / s_{P, V}\right) \leq S(r, w)$. Let $z_{0}$ be a zero of $V(z)$ which is neither a zero of $c(z)$ nor a zero of coefficients of $P(z, w)$ as well as coefficients of $V_{j}(z, w)(j=1, \ldots, k)$ nor a zero of $s_{V, V_{z}}(z)$ and $s_{P, V}(z)$. By the proof of Theorem $1, w^{\prime}\left(z_{0}\right)=0$ and hence $\left(V^{\prime}(z)=d V(z, w(z)) / d z\right.$ and $\left.V_{z}(z)=\partial V(z, w) /\left.\partial\right|_{w=w(z)}\right)$

$$
V^{\prime}\left(z_{0}\right)=V_{z}\left(z_{0}\right)+w^{\prime}\left(z_{0}\right) V_{w}\left(z_{0}, w\left(z_{0}\right)\right)-V_{z}\left(z_{0}\right) \neq 0 \text {. }
$$

Thus $z_{0}$ is a simple zero of $V(z)$. By Lemma $A(b), z_{0}$ is a double pole of $\{w, z\}$. Thus by $(1.1), 2 m=\mu_{j_{0}}$.

(ii) We may write $V_{j_{1}}(z, w)=w-\tau, \tau \in \mathbb{C}$. By Lemma 1 and Remark $1, m(r, \tau ; w)=S(r, w)$ and $N\left(r, 0 ; P, V_{j_{1}}\right) \leq N\left(r, 1 / s_{P, v_{j_{1}}}\right) \leq S(r, w)$. Let $z_{0}$ be a $\tau$-point of $w$ which is neither a pole nor a zero of $c(f)$, neither a pole nor a zero of coefficients of $P(z, w)$ and $V_{j}(z, w), j=1, \ldots, k$, nor a zero of $s_{P, v_{j_{1}}}(z)$. Since $\{w, z\}$ has a pole at $z_{0}$, we must have $\omega\left(z_{0}, 1 /(w-\tau)\right)=n \geq 2$. Thus $z_{0}$ is a double pole of $\{w, z\}$, and hence

$$
2 m=n \mu_{j_{1}} \text {, }
$$

which implies that $\mu_{j_{1}} \leq m$ and $\mu_{j_{1}} \mid(2 m)$.

Proof of Theorem 2. The following four cases are to be considered.

I. There are $V_{1}(z, w) \neq V_{2}(z, w)$ such that $V_{1 z}(z) V_{2 z}(z) \neq 0$. 
II. There is only one $V_{1}(z, w)$ for which $V_{1 z}(z) \neq 0$. Further we suppose that $\operatorname{deg}_{w}\left[V_{1}(z, w)\right] \geq 2$.

III. There is only one $V_{1}(z, w)$ for which $V_{1 z}(z) \not \equiv 0$. Further we suppose that $\operatorname{deg}_{w}\left[V_{1}(z, w)\right]=1$.

IV. $V_{j z}(z) \equiv 0$ for any $j$.

We will treat these four cases in order.

CASE I. By Lemma 2 and Theorem $1, Q(z, w)$ is of the form (1.4), since $d \leq 4 m$.

CASE II. By Lemma 2 and Theorem $1, Q(z, w)$ is of the form (1.5), since $d \leq 4 m$.

CASE III. $Q(z, w)$ must be of the form

$$
Q(z, w)=c(z)(w+b(z))^{2 m}\left(w-\tau_{1}\right)^{\mu_{1}} \cdots\left(w-\tau_{k}\right)^{\mu_{k}},
$$

where $\tau_{1}, \ldots, \tau_{k}$ are distinct constants. By Lemma 1 and (3.6) we have

$$
\begin{aligned}
2 m \bar{N}\left(r, 1 / w^{\prime}\right)= & 2 m N\left(r, \frac{1}{w+b(z)}\right) \\
& +\sum_{j=1}^{k} \mu_{j} N\left(r, \tau_{j} ; w\right)+S(r, w) \\
= & \left(2 m+\sum_{j=1}^{k} \mu_{j}\right) T(r, w)+S(r, w) .
\end{aligned}
$$

Let $z_{j}$ be a $\tau_{j}$-point of $w(z)$ such that $\xi_{t}\left(z_{j}\right) \neq 0, \infty, \eta_{i}\left(z_{j}\right) \neq 0, \infty$, for $t=0, \ldots, p, i=0, \ldots, q$ and $s_{P, V}\left(z_{j}\right) \neq 0$, where $V_{j}=w-\tau_{j}$. Let $\omega\left(z_{j}, 1 /\left(w-\tau_{j}\right)\right)=n_{j} \geq 2$. By $(4.1)$

$$
2 m=n_{j} \mu_{j} .
$$

Since $\omega\left(z_{j}, 1 / w^{\prime}\right)=n_{j}-1$, we have by Lemma 1 that

$$
\begin{aligned}
N_{1}\left(r, 0 ; w^{\prime}\right) & \geq \sum_{j=1}^{k}\left(\frac{n_{j}-2}{n_{j}}\right) N\left(r, \tau_{j} ; w\right)+S(r, w) \\
& \geq\left(k-2 \sum_{j=1}^{k} \frac{1}{n_{j}}\right) T(r, w)+S(r, w)
\end{aligned}
$$

We have

$$
\begin{aligned}
2 T(r, w) & \geq N\left(r, 1 / w^{\prime}\right)+S(r, w) \\
& \geq \bar{N}\left(r, 1 / w^{\prime}\right)+N_{1}\left(r, 1 / w^{\prime}\right)+S(r, w)
\end{aligned}
$$


By (4.2)-(4.5) we have

$$
\begin{aligned}
2 T(r, w) \geq & \left(1+\sum_{j=1}^{k} \frac{\mu_{j}}{2 m}\right) T(r, w) \\
& +\left(k-2 \sum_{j=1}^{k} \frac{\mu_{j}}{2 m}\right) T(r, w)+S(r, w),
\end{aligned}
$$

that is,

$$
\left(1+\sum_{j=1}^{k} \frac{\mu_{j}}{2 m}\right) T(r, w) \geq k T(r, w)+S(r, w),
$$

and hence we obtain

$$
1+\sum_{j=1}^{k} \frac{\mu_{j}}{2 m} \geq k
$$

By Theorem 1, $2 m+\sum_{j=1}^{k} \mu_{j} \leq 4 m, \sum_{j=1}^{k} \mu_{j} \leq 2 m$. Therefore $k \leq 2$.

If $k=2$, then $\mu_{1}+\mu_{2}=2 m$ and $\mu_{1}=\mu_{2}=m$ by Lemma 2(ii). Thus we obtain (1.7).

If $k=1$, then we obtain (1.8). If $k=0$, then we obtain (1.6).

Case IV. $Q(z, w)$ must be of the form

$$
Q(z, w)=c(z)\left(w-\tau_{1}\right)^{\mu_{1}} \cdots\left(w-\tau_{k}\right)^{\mu_{k}},
$$

where $\tau_{1}, \ldots, \tau_{k}$ are distinct constants. By Lemma 1

$$
\begin{aligned}
2 m \bar{N}\left(r, 1 / w^{\prime}\right) & =\sum_{j=1}^{k} \mu_{j} N\left(r, \tau_{j} ; w\right)+S(r, w) \\
& =\sum_{j=1}^{k} \mu_{j} T(r, w)+S(r, w) .
\end{aligned}
$$

By (4.3)-(4.5) we obtain, as in the case III, that

$$
2+\sum_{j=1}^{k} \frac{\mu_{j}}{2 m} \geq k .
$$

By Theorem 1, $\sum_{j=1}^{k} \mu_{j} \leq 4 m$. Hence we get $k \leq 4$.

If $k=4$, then $\mu_{1}=\mu_{2}=\mu_{3}=\mu_{4}=m$ by Lemma 2(ii), and we have (1.9).

If $k=3$, then from (4.7) and (4.3)

$$
\frac{\mu_{1}+\mu_{2}+\mu_{3}}{2 m}=\frac{1}{n_{1}}+\frac{1}{n_{2}}+\frac{1}{n_{3}} \geq 1 \quad\left(n_{j} \geq 2\right) .
$$


The only triplets $\left(n_{1}, n_{2}, n_{3}\right)$ which satisfy (4.8) are as follows (we suppose $\left.n_{1} \leq n_{2} \leq n_{3}\right)$ :

$$
\left\{\begin{array}{l}
(2,2, n), \text { where either } n=2, \text { or } n \geq 3 \text { and } n \mid m ; \\
(2,3,3),(2,3,4),(2,3,5),(2,3,6) \\
(2,4,4),(3,3,3)
\end{array}\right.
$$

Therefore we get, by (4.9), that

$$
\left.\left(\mu_{1}, \mu_{2}, \mu_{3}\right)=(m, m, 2 m / n) \text { (either } n=2 \text {, or } n \geq 3 \text { and } n \mid m\right) \text {, }
$$
which corresponds to $(1.10)$;

$\left(\mu_{1}, \mu_{2}, \mu_{3}\right)=(m, 2 m / 3,2 m / 3)$ which corresponds to (1.11);

$\left(\mu_{1}, \mu_{2}, \mu_{3}\right)=(m, 2 m / 3,2 m / 4)$ which corresponds to (1.12);

$\left(\mu_{1}, \mu_{2}, \mu_{3}\right)=(m, 2 m / 3,2 m / 5)$ which corresponds to (1.13);

$\left(\mu_{1}, \mu_{2}, \mu_{3}\right)=(m, 2 m / 3,2 m / 6)$ which corresponds to (1.14);

$\left(\mu_{1}, \mu_{2}, \mu_{3}\right)=(m, 2 m / 4,2 m / 4)$ which corresponds to $(1.15)$;

$\left(\mu_{1}, \mu_{2}, \mu_{3}\right)=(2 m / 3,2 m / 3,2 m / 3)$ which corresponds to $(1.16)$.

If $k=2$, then $n_{j} \mid m$ and $n_{j} \geq 2(j=1,2)$, and we get (1.17).

If $k=1$, then we have (1.18). If $k=0$, we obtain (1.19).

\section{Proofs of Lemma 3, 4, 5, 6, 7, 8, and Theorem 3}

We suppose that the equation (1.1) is of the form (1.20).

Proof of Lemma 3. Let $z_{i}$ be a $\sigma_{i}$ point of $w(z)$. Then $z_{i}$ is a zero of $\{w, z\}$, and hence by Lemma $\mathbf{A}(\mathbf{b}), z_{i}$ is not a zero of $w^{\prime}(z)$. Thus $\omega\left(z_{i}, 1 /\left(w-\sigma_{i}\right)\right)=1$. Put $\omega\left(z_{i}, 1 /\{w, z\}\right)=n$. Then

$$
n m=\lambda_{i} \text {, and hence } m \mid \lambda_{i} \text {. }
$$

Proof of Lemma 4. Suppose that $n \geq 3$ in (1.18). We have that $h=1$ in (1.20). In fact, suppose $h \geq 2$. Since $\operatorname{deg}[P(w)]=\operatorname{deg}[Q(w)]=2 m / n<m$, we have that $\sigma_{1}$ and $\sigma_{2}$ are Picard exceptional values of $w(z)$ by Lemma 3. Thus, by Theorem $1, d=\operatorname{deg}[Q(w)]=0$, which is a contradiction. Thus we may assume that the equation is of the following form:

$$
\{w, z\}^{m}=c_{0}\left(\frac{w-\sigma}{w-\tau}\right)^{2 m / n}, \quad c_{0}(\neq 0) \in \mathbb{C} .
$$

Put $u=(w-\sigma) /(w-\tau)$ in (5.2). Then by Lemma A(c),

$$
\{u, z\}^{n}=c u^{2}, \quad c=c_{0}^{n / m} \text {. }
$$


By the lemma on logarithmic derivatives,

$$
2 m(r, u)+O(1)=m\left(r, c u^{2}\right)=m\left(r,\{u, z\}^{n}\right)=S(r, u),
$$

and hence

$$
m(r, u)=S(r, u) .
$$

Let $z_{0}$ be a pole of $u(z)$ and $\omega\left(z_{0}, u\right)=\mu$. Suppose $\mu=1$. Then by Lemma $\mathrm{A}(\mathrm{a}),\{u, z\}$ is regular at $z_{0}$, which contradicts (5.3). Thus $\mu \geq 2$. By Lemma $\mathrm{A}(\mathrm{b}), 2 n=2 \mu$. Therefore $u$ has infinitely many poles of order $n$. By Lemma $3, u$ has no zeros since $n \geq 3$ and hence $n \nmid 2$. Also $u^{\prime}$ has no zeros as seen by Lemma $\mathrm{A}(\mathrm{b})$. Thus $u^{\prime} / u$ and $u^{\prime \prime} / u^{\prime}$ admit (simple) poles at poles of $u$ only. Further, residues of $u^{\prime} / u$ and $u^{\prime \prime} / u^{\prime}$ are $-n$ and $-(n+1)$, respectively. If we put

$$
n \phi=(n+1) u^{\prime} / u-n u^{\prime \prime} / u^{\prime},
$$

then $\phi$ is an entire function. We have

$$
m(r, \phi)=S(r, u),
$$

and hence $\phi$ is a small function for $u$.

Write $u^{\prime} / u=f$ and $u^{\prime \prime} / u^{\prime}=g$. Then

$$
f^{\prime}=f g-f^{2} \text {. }
$$

From (5.5) we have

$$
g=a f-\phi,
$$

where $a=(n+1) / n$. From (5.7) and (5.8) we have

$$
g^{\prime}=a f^{\prime}-\phi^{\prime}=\left(a^{2}-a\right) f^{2}-a \phi f-\phi^{\prime} .
$$

From (5.9) and (5.8) we obtain

$$
\{u, z\}=g^{\prime}-\frac{1}{2} g^{2}=\left(a^{2} / 2-a\right) f^{2}-\phi^{\prime}-\frac{1}{2} \phi^{2} .
$$

Since we supposed $n \geq 3$, we get $A=a^{2} / 2-a=a(a-2) / 2 \neq 0$. Thus $u$ satisfies the first order differential equation

$$
\left(A\left(u^{\prime} / u\right)^{2}-\Phi\right)^{n}=c u^{2} \text {, }
$$

where $\Phi=\phi^{\prime}+\frac{1}{2} \phi^{2}$. Now $\Phi \not \equiv$ since, if $\Phi \equiv 0$, then equation (5.10) does not admit a transcendental solution. Put $F=A f^{2}-\Phi=A\left(u^{\prime} / u\right)^{2}-\Phi$. Then $F$ has no zeros as seen from (5.10), since $u$ has no zeros. Applying Theorem B to $f=u^{\prime} / u$, noting $\Phi$ is a small function with respect to $f(z)$, we obtain

$$
\begin{aligned}
2 T(r, f) & \leq \bar{N}(r, f)+\bar{N}(r, 0 ; f)+\bar{N}(r, 0 ; F)+S(r, f) \\
& \leq \bar{N}(r, f)+S(r, f) \leq T(r, f)+S(r, f)
\end{aligned}
$$


a contradiction, which shows that $n<3$.

Proof of Lemma 5. Since we are assuming $\operatorname{deg}[P]=\operatorname{deg}[Q]$, the case (1.19), that is, $Q(w)=C$, a constant, need not to be considered. Since $Q$ is independent of $z$, only (1.9) $-(1.18)$ are to be considered. It suffices to show that the solution $w(z)$ takes any $\sigma_{i}$, as seen by Lemma 3. To the contrary we suppose that $w(z)$ has no $\sigma_{i}$ points for some $i$. By Theorem $1, d \leq 2 m$. This is impossible for (1.9)-(1.13), since $p=q$. If $Q(w)$ is of the form (1.14), by Lemma 1 , we have $m\left(r, \tau_{j} ; w\right)=S(r, w), j=1,2,3$. Let $z_{j}$ be a $\tau_{j}$ point. Then by (4.3), $\omega\left(z_{1}, 1 / w-\tau_{1}\right)=2, \omega\left(z_{2}, 1 / w-\tau_{2}\right)=$ $3, \omega\left(z_{3}, 1 / w-\tau_{3}\right)=6$. Hence we have $\sum_{j=1}^{3} \theta\left(\tau_{j}, w\right)=1 / 2+2 / 3+$ $5 / 6=2$, which contradicts Nevanlinna's theorem on total ramification, since $w(z)$ is supposed to omit $\sigma_{i}$. Similarly to the case (1.14), if $Q(w)$ is of the form (1.15) and (1.16), then we have $\sum_{j=1}^{3} \theta\left(\tau_{j}, w\right)=2 / 3+2 / 3+$ $2 / 3=2$, and $\sum_{j=1}^{3} \theta\left(\tau_{j}, w\right)=1 / 2+3 / 4+3 / 4$, respectively, which are also contradictions. Thus for (1.9)-(1.13) and (1.14)-(1.16), $w(z)$ must take $\sigma_{i}$, $i=1,2, \ldots, h$.

Suppose $Q(w)$ is of the form (1.17). If $n_{1}>2$ or $n_{2}>2$, then similarly to the case (1.14), we have $\theta\left(\tau_{1}, w\right)+\theta\left(\tau_{2}, w\right)=\left(n_{1}-1\right) / n_{1}+\left(n_{2}-1\right) / n_{2} \geq$ $7 / 6 /$, which contradicts Nevanlinna's theorem, and since we have $n_{1}=n_{2}=$ 2. Suppose $P(w)$ has a factor $(w-\sigma)^{\lambda}, m+\lambda$. Since $p=q=2 m$ in (1.17), there is another factor $(w-\tilde{\sigma})^{\tilde{\lambda}}, m+\tilde{\lambda}$. Then both $\sigma$ and $\tilde{\sigma}$ are Picard values for $w(z)$, and we have $d=0$ by Theorem 1 , which is a contradiction.

Finally suppose $Q(w)$ is of the form (1.18). By Lemma 4, $\operatorname{deg}[Q]=$ $m$. As in (1.17), we see that $P(w)$ must be of the form $(w-\sigma)^{m}$, which completes the proof of Lemma 5.

Proof of Lemma 6. Suppose (2.5) possesses an admissible solution $w(z)$. Put $u=1 /\left(w-\tau_{3}\right)$. Then by Lemma A(c), we have

$$
\{u, z\}=C\left(u-s_{1}\right)\left(u-s_{2}\right)\left(u-s_{3}\right) /\left[\left(u-t_{1}\right)\left(u-t_{2}\right)\right] .
$$

By Lemma 1 and (4.3), $w(z)$ has infinitely many $\tau_{3}$ points which are all of multiplicity 2 . Therefore $u(z)$ has infinitely many poles which are all of order 2 . Therefore $u(z)$ has infinitely many poles which are all of order 2 . Let $z_{0}$ be a pole of $u(z)$, then

$$
u(z)=\frac{R}{\left(z-z_{0}\right)^{2}}+\frac{\alpha}{\left(z-z_{0}\right)}+O(1), \quad R \neq 0 .
$$

By Lemma $A(b)$, we get

$$
\{u, z\}=\frac{-3 / 2}{\left(z-z_{0}\right)^{2}}+\frac{3 \alpha / 2 R}{\left(z-z_{0}\right)}+O(1) .
$$


On the other hand, the right-hand side of (5.11) can be written

$$
\begin{aligned}
& C\left(u-s_{1}\right)\left(u-s_{2}\right)\left(u-s_{3}\right) /\left[\left(u-t_{1}\right)\left(u-t_{2}\right)\right] \\
& =C R\left(z-z_{0}\right)^{-2}+C \alpha\left(z-z_{0}\right)^{-1}+O(1)
\end{aligned}
$$

near $z_{0}$. Thus, from (5.13) and (5.14), $-3 / 2=C R$ and $3 \alpha / 2 R=C \alpha$. Hence

$$
R=-\frac{3}{2 C}, \quad \alpha=0
$$

Put

$$
H(z)=u^{\prime}(z)^{2} /\left[\left(u(z)-t_{1}\right)\left(u(z)-t_{2}\right)\right] .
$$

By (4.3), each $t_{j}$ point $(j=1,2)$ is of multiplicity 2 . Thus $u^{\prime}(z)$ has a simple zero there. Hence $H(z) \neq \infty$ at $t_{j}$ points of $u(z)$. Thus, if $z_{0}$ is pole of $H(z)$, then $z_{0}$ is pole of $u(z)$, and $\omega\left(z_{0}, H\right)=2$. By (5.12) and (5.15) we have

$$
H(z)=4\left(z-z_{0}\right)^{-2}+O(1)
$$

Put

$$
\varphi(z)=\frac{H(z)}{4}+\frac{2 C}{3} u(z) .
$$

Then by (5.12), (5.15) and (5.16), $\varphi(z)$ is regular at $z_{0}$. Thus $\varphi(z)$ is an entire function. By Lemma 1, we have

$$
m(r, u)=m\left(r, \tau_{3} ; w\right)=S(r, w)=S(r, u) .
$$

Hence by the lemma on logarithmic derivatives

$$
\begin{aligned}
m(r, \varphi) \leq & m(r, H)+m(r, u)+O(1) \\
\leq & m\left(r, u^{\prime} /\left(u-\tau_{1}\right)\right)+m\left(r, u^{\prime} /\left(u-\tau_{2}\right)\right) \\
& +m(r, u)+O(1)=S(r, u) .
\end{aligned}
$$

Therefore $\varphi(z)$ is a small function for $u(z)$. From (5.17), we have

$$
u^{\prime 2}=C^{*}\left(u-t_{1}\right)\left(u-t_{2}\right)(u-\tilde{\varphi}), \quad C^{*}=-8 C / 3, \quad \tilde{\varphi}=-3 \varphi / 2 C .
$$

By Theorem $\mathrm{C}$ and (5.18), $\tilde{\varphi}$ is a constant.

Let $z_{*}$ be a zero of $u^{\prime}(z)$. By Lemma $\mathrm{A}(\mathrm{b}), z_{*}$ is a pole of $\{u, z\}$, whence $z_{*}$ is a $t_{1}$ or $t_{2}$ point of $u(z)$, as seen from (5.11). Thus, zeros of $u^{\prime}$ are $t_{j}$ points of $u$, therefore by (5.18) we have that $\tilde{\varphi}=t_{1}$ or $t_{2}$, or $\tilde{\varphi}$ is Picard exceptional value. If $\tilde{\varphi}=t_{1}$, then

$$
u^{\prime 3}=C^{*}\left(u-t_{1}\right)^{2}\left(u-t_{2}\right) \text {. }
$$

Suppose $u\left(z_{1}\right)=t_{1}$ and put $\omega\left(z_{1}, 1 /\left(u-t_{1}\right)\right)=l$. Then $\omega\left(z_{1}, 1 / u^{\prime 2}\right)=$ $(2 l-2) \neq \omega\left(z_{1}, 1 / C^{*}\left(u-t_{2}\right)\right)=2 l$, whence $u(z)$ cannot take $t_{1}$. This 
contradicts Lemma 1 , and hence $\tilde{\varphi} \neq t_{1}$. Similarly $\tilde{\varphi} \neq t_{2}$. If $\tilde{\varphi}$ is a Picard exceptional value, then $2 \geq d=3$ by Theorem 1 . Hence we obtain a contradiction. Therefore (5.11), and hence (2.5), cannot possess admissible solutions.

Proof of Lemma 7. Suppose (2.6) possesses an admissible solution $w(z)$. Put $u=c(w-\sigma) /(w-\tau)$. Then

$$
\{u, z\}=u \text {. }
$$

By the lemma on the logarithmic derivative, $m(r, u)=m(r,\{u, z\})=$ $S(r, u)$. Hence $u(z)$ has infinitely many poles, which are of multiplicity 2 by (5.19) and Lemma $\mathrm{A}(\mathrm{b})$. Let $z_{0}$ be a pole of $u(z)$. Then

$$
u(z)=\frac{R}{\left(z-z_{0}\right)^{2}}+\frac{\alpha}{\left(z-z_{0}\right)}+O(1) \quad(R \neq 0) .
$$

Arguing as in the proof of Lemma 6, we obtain

$$
R=3 / 2, \quad \alpha=0 .
$$

Put $g=u^{\prime \prime} / u^{\prime}$ in (5.19). Then

$$
g^{\prime}-\frac{1}{2} g^{2}=u .
$$

By (5.19) and Lemma A(b), we see that $u^{\prime}$ has no zeros. Thus if $\tilde{z}$ is a pole of $g(z)$, then $\tilde{z}$ is a pole of $u(z)$. From (5.20) and $\alpha=0$, we get

$$
g(z)=-3 /\left(z-z_{0}\right)+O\left(z-z_{0}\right) .
$$

Put

$$
\varphi=g^{\prime}-\frac{1}{3} g^{2} .
$$

Then $\varphi(z)$ is regular at $z_{0}$. Thus $\varphi(z)$ is entire. On the other hand

$$
\begin{aligned}
T(r, g) & =m(r, g)+N(r, g)=m\left(r, u^{\prime \prime} / u^{\prime}\right)+\bar{N}(r, u) \\
& =\frac{1}{2} T(r, u)+S(r, u),
\end{aligned}
$$

which shows that $S(r, u)=S(r, g)$. Thus

$$
m(r, \varphi) \leq m(r,\{u, z\})+m\left(r,\left(u^{\prime \prime} / u^{\prime}\right)^{2}\right)+O(1)=S(r, g) .
$$

Thus $\varphi(z)$ is a small function for $g(z)$. From (5.22) and (5.24), we have

$$
\varphi(z)-\frac{1}{6} g(z)^{2}=u(z) .
$$


By (5.26) and (5.24),

$$
\begin{aligned}
u^{\prime} & =\varphi^{\prime}-\frac{1}{3} g g^{\prime}=\varphi^{\prime}-\frac{1}{9} g^{3}-\frac{1}{3} \varphi g, \\
u^{\prime \prime} & =\varphi^{\prime \prime}-\frac{1}{3} g^{\prime 2}-\frac{1}{3} g g^{\prime \prime} \\
& =\varphi^{\prime \prime}-\frac{1}{3} g^{\prime 2}-\frac{1}{3} g\left(\varphi^{\prime}+\frac{2}{3} g g^{\prime}\right) \\
& =\varphi^{\prime \prime}-\frac{1}{3} \varphi^{2}-\frac{1}{9} g^{4}-\frac{4}{9} \varphi g^{2}-\frac{1}{3} \varphi^{\prime} g .
\end{aligned}
$$

Hence

$$
g=u^{\prime \prime} / u^{\prime}=\frac{9 \varphi^{\prime \prime}-3 \varphi^{2}-g^{4}-4 \varphi g^{2}-3 \varphi^{\prime} g}{9 \varphi^{\prime}-g^{3}-3 \varphi g}
$$

that is,

$$
\varphi g^{2}+12 \varphi^{\prime} g-9 \varphi^{\prime \prime}+3 \varphi^{2}=0 .
$$

if $\varphi \not \equiv 0, g$ must be small for $g$, as seen by solving the quadratic equation (5.27) for $g$. Thus is impossible, and hence $\varphi \equiv 0$. Therefore by (5.24), $g(z)$ cannot be transcendental. This shows that $u$ and hence $w$ is not transcendental, contrary to our hypothesis.

Proof of Lemma 8. Suppose (2.7) possesses an admissible solution $w(z)$. By Lemma 5 , we have $\sigma_{1}=\sigma_{2}$. Let $L$ be a Möbius transformation which maps $\sigma_{1}, \tau_{1}, \tau_{2}$ to $\infty, 1,-1$, respectively. Put $u=L(w)$. Then

$$
\{u, z\}^{2}=1 /\left(1-u^{2}\right) \text {. }
$$

Put $V(z)^{2}=v(z)=1 /\left(1-u(z)^{2}\right)$. Then $V(z)$ is meromorphic by (5.28) and by a simple calculation, we obtain

$$
\left[\{v, z\}+\frac{3}{8}\left(\frac{v^{\prime}}{v(v-1)}\right)^{2}\right]^{2}=V^{2}=v .
$$

By Lemma 1 and (5.28), $u(z) \pm 1$ have infinitely many zeros, which are of multiplicity 4 by (4.3). Thus poles of $v(z)$ are infinite in number and of order 4. Let $z_{0}$ be a pole of $v(z)$. Then

$$
v(z)=\frac{R}{\left(z-z_{0}\right)^{4}}+\frac{\alpha}{\left(z-z_{0}\right)^{4}}+O\left(\left(z-z_{0}\right)^{-2}\right), \quad R \neq 0 .
$$

Also $\frac{v^{\prime}}{v(v-1)}$ is regular at $z_{0}$, and thus we get by Lemma $\mathrm{A}(\mathrm{b})$

$$
\{v, z\}+\frac{3}{8}\left(\frac{v^{\prime}}{v(v-1)}\right)^{2}=\frac{-15 / 2}{\left(z-z_{0}\right)^{2}}+\frac{15 \alpha / 4 R}{\left(z-z_{0}\right)}+O(1)
$$


From (5.30) and (5.31), $R=(-15 / 2)^{2}, \alpha=-(-15 / 2)^{2} \alpha / R$. Hence we have

$$
R=225 / 4, \quad \alpha=0 .
$$

From (5.29) we see that, if $v^{\prime}(\tilde{z})=0$, then $v(\tilde{z})=0$ or 1 , since $v$ is regular and $\{v, z\}=\infty$ at $z=\tilde{z}$. If $v(\tilde{z})=0$, then $u(\tilde{z})=\infty$. By (5.28) and Lemma $\mathrm{A}(\mathrm{a}), \tilde{z}$ is a simple pole of $u$ and hence is a double zero of $v$. If $v(\tilde{z})=1$, then $u(\tilde{z})=0$. By $(5.28), u^{\prime}(\tilde{z}) \neq 0$. Thus $\tilde{z}$ is a simple zero of $u$ and hence a double zero of $v(z)-1$. Hence $\omega\left(\tilde{z}, 1 / v^{\prime}\right)=1$. If we put

$$
h=v^{\prime 2} /[v(v-1)], \text { and } \phi=h^{\prime} / h,
$$

then $h(z)$ has no zeros and infinitely many poles only at poles of $v$. From (5.33), poles of $h$ are of order 2. Hence by (5.30) and (5.32) we have

$$
h(z)=\frac{16}{\left(z-z_{0}\right)^{2}}+O(1) .
$$

Since $h(z)$ has no zeros, $\phi=\infty$ only at poles of $h$, and hence at poles of $v$. Thus by (5.34), we can write $\phi$ as

$$
\phi(z)=\frac{-2}{\left(z-z_{0}\right)}+O\left(z-z_{0}\right) .
$$

Put

$$
\tau(z)=\phi^{\prime}(z)-\frac{1}{2} \phi(z)^{2} \text { and } \sigma(z)=\phi^{\prime}(z)-\frac{1}{8} h(z) .
$$

Then $\tau(z)$ and $\sigma(z)$ are regular at $z_{0}$. Thus $\tau(z)$ and $\sigma(z)$ are entire. By Lemma 1 and (5.29), (5.33), we have

$$
\begin{aligned}
2 T(r, V)+O(1) & =T(r, v)=m(r, v)+N(r, v) \\
& =N(r, v)+S(r, u)
\end{aligned}
$$

and

$$
\begin{aligned}
T(r, h) & =m(r, h)+N(r, h)=S(r, v)+\frac{1}{2} N(r, v) \\
& =\frac{1}{2} T(r, v)+S(r, v) .
\end{aligned}
$$

Hence $S(r, u)=S(r, v)=S(r, V)=S(r, h)$. From (5.36) and (5.33),

$$
m(r, \tau) \leq m\left(r,\left(h^{\prime \prime} / h\right)-\left(h^{\prime} / h\right)^{2}\right)+m\left(r,\left(h^{\prime} / h\right)^{2}\right)+O(1)=S(r, V),
$$

and

$$
m(r, \sigma) \leq m\left(r,\left(h^{\prime \prime} / h\right)-\left(h^{\prime} / h\right)^{2}\right)+m(r, h)+O(1)=S(r, V) .
$$

Thus, $\tau(z)$ and $\sigma(z)$ are small functions for $V(z)$. From (5.29) and (5.33), we have by simple calculation

$$
V=\frac{1}{2} \phi^{\prime}-\frac{1}{8} \phi^{2}-\frac{1}{2} h .
$$


From (5.37) and (5.36), we have

$$
V=(-15 / 32) h+\kappa,
$$

where $\kappa(z)=\frac{1}{4}(\tau(z)+\sigma(z))$. On the other hand, from (5.29) and (5.33) we have

$$
h=\left(2 V V^{\prime}\right)^{2} /\left[V^{2}\left(V^{2}-1\right)\right]=4 V^{\prime 2} /\left(V^{2}-1\right) .
$$

Hence, from (5.38) and (5.39), $V(z)$ satisfies the equation

$$
V^{\prime 2}=-\frac{8}{15}(V-\kappa)(V-1)(V+1) .
$$

By Theorem C and (5.40), $\kappa(z)$ is a constant. If $\kappa \neq \pm 1$, then $V(z)-\kappa$ has infinitely many double zeros, and hence $v^{\prime}(z)=0$ at some (infinitely many) zeros of $v-\kappa^{2}$, which is impossible by (5.29). Therefore $\kappa=1$ or -1 .

By $(5.40), V^{\prime \prime}=-\frac{4}{15}\left(3 V^{2}-2 \kappa V-1\right)$. Since $V^{2}=1 /\left(1-u^{2}\right)$, we get $\left(u^{\prime} / u\right)^{2}=V^{\prime 2}\left[V\left(V^{2}-1\right)\right]^{2}=-\frac{8}{15}(V-\kappa) /\left[V^{2}\left(V^{2}-1\right)\right]$ and $u^{\prime \prime} / u=$ $-2 V^{\prime} / V+\frac{1}{2} V^{\prime} /(V-\kappa)$. thus we obtain

$$
\{u, z\}=-2 V^{\prime \prime} / V+\frac{1}{2} V^{\prime \prime} /(V-\kappa)-\frac{5}{8} V^{\prime 2} /(V-\kappa)^{2}+V^{\prime 2} /[V(V-\kappa)] .
$$

Using (5.40), we have that

$$
\begin{aligned}
& \{u, z\}=V+\frac{13}{15}, \quad\left(\{u, z\}-\frac{13}{15}\right)^{2}=V^{2}=1 /\left(1-u^{2}\right) \quad \text { if } \kappa=1, \\
& \{u, z\}=V-\frac{13}{15}, \quad\left(\{u, z\}-\frac{13}{15}\right)^{2}=V^{2}=1 /\left(1-u^{2}\right) \quad \text { if } \kappa=1,
\end{aligned}
$$

which contradicts $(5.28)$. Therefore, (2.7) cannot possess any admissible solutions.

Proof of Theorem 3. Suppose the equation (1.20) possesses an admissible solution $w=w(z)$. We assume that $p=q=d$, by applying a Möbius transformation if necessary. By Lemma $5, q=p$ is a multiple of $m$. Thus $Q(w)$ cannot be the form of (1.11)-(1.13). If $Q(w)$ is of the form of (1.10), then $n=2$ by the same reason. In this case, the equation (1.20) must be the form of (2.5), which is impossible by Lemma 6 . If $Q(w)$ is the form of (1.18), then (1.20) is the form of (2.6), which is also rejected by Lemma 7. If $Q(w)$ is the form of (1.17), then by Lemma 5 we have

$$
2 m / n_{1}+2 m / n_{2}=2 m \text { or } m \quad\left(n_{1}, n_{2} \geq 2\right) \text {. }
$$

Thus $\left(n_{1}, n_{2}\right)=(2,2)$ or $(4.4)$. Therefore we get the form of $(1.25)$ or (2.7), respectively. By Lemma 8 , the case $(4,4)$ is rejected. If $Q(w)$ is of the 
form of (1.9), (1.14), (1.15), (1.16) or (1.19), then we obtain the equation of the form (1.21), (1.22), (1.23), (1.24) or (1.26), respectively, which proves our assertion.

\section{References}

[1] W. K. Hayman, Meromorphic functions, (Oxford University Press, 1964).

[2] E. Hille, Ordinary differential equations in the complex domain, (Wiley-Interscience, New York, 1976).

[3] K. Ishizaki, 'On some generalizations of theorems of Toda and Weissesnborn to differential polynomials', Nagoya Math. J. 115 (1989), 199-207.

[4] K. Ishizaki, 'Deficiencies of the admissible solutions of the Schwarzian differential equations and the Ricatti equations', Res. Rep. Tokyo Nat. College Tech. 201988.

[5] G. Jank and L. Volkmann, Meromorphe Funktionen und Differential-geichungen, (Birk häuser-Verlag, Basel, Boston, Stuttgart, 1985).

[6] I. Laine, 'On the behaviour of the solutions of some first order differential equations', Ann. Acad. Sci. Fenn. Ser. A I, 497 (1971), 1-26.

[7] A. Z. Mokhon'ko, 'On the Nevanlinna characteristics of certain meromorphic functions' (Russian). Teor. Funkcii Funkcional. Anal. i Priložen. 14 (1971), 83-87.

[8] E. Mues, 'Uber faktorisierbare Lösungen von Riccatischen Differentialgeichungen', Math. Z. 121 (1971), 145-156.

[9] R. Nevanlinna, Analytic functions, (Springer-Verlag, Berlin, Heidelberg, New York, 1970).

[10] J. V. Rieth, Untersuchungen Gewisser Klassen Gewöhnlicher Differentialgleichungen erster und zweiter ordnung im Komplexen, (Doctoral Dissertation, Technische Hochschule, Aachen, 1986).

[11] N. Steinmetz, 'On factorization of the solutions of the Schwarzian differential equation $\{w, z\}=q(z)^{\prime}$, Funkcial Ekvac. 24 (1981), 307-315.

[12] He Yuzan and I. Laine, 'The Hayman-Miles theorem and the differential equation $\left(y^{\prime}\right)^{n}=R(z, y)$, to appear.

Department of Mathematics

Tokyo National College of Technology

1220 -2 Kunugida-cho

Hachioji

Tokyo 193

Japan 